\title{
Postestructuralismo y ESTÉTICA: ENTRE ARTE Y GÉNERO
}

\author{
Naim Garnica ${ }^{1}$
}

RESUmen: El objetivo general del trabajo es analizar las influencias y recepciones del post-estructuralismo en las lecturas de Judith Butler y sólo algunas derivaciones en el pensamiento de Paul B. Preciado. A partir de este rastreo se pueden establecer las críticas de la teoría queer al feminismo a través de las imágenes y textualidades que propone el arte. La hipótesis general del trabajo supone que, a partir de la recepción del postestructuralismo en los análisis de Butler y Preciado, se podría reconocer en la estética la capacidad crítica y política que las teorías de la identidad sexual pretenden lograr. A los efectos de identificar estos legados nos detendremos en reconocer los aportes de Jacques Derrida en la obra de estxs autorxs. Para finalizar dejamos planteadas algunas críticas que podrían advertir sobre los posibles límites en el plano de la estética de las teorías de género.

Palabras Claves: Estética. Género. Crítica. Arte. Danza.

\section{INTRODUCCIÓN}

A partir del surgimiento del postestructuralismo aparecen en el campo de la filosofía perspectivas que abordan los cuerpos en torno a cuestiones de género y sexualidad. Las influencias post-estructuralistas en las críticas a los feminismos clásicos, nos invitan a reflexionar acerca de cómo deconstruimos las categorías: cuerpo, género y sexualidad. Entre ellas encontramos las teorías queer, nacidas en el texto: Gender trouble de Judith Butler, el cual mediante sus criticas originó textos como El manifiesto contra-sexual o Manifiesto para cyborgs, de Beatriz Preciado y Donna Haraway respectivamente. Siguiendo estas reflexiones la teoría queer busca descomponer la hegemonía con la que pensamos la construcción de las subjetividades. En este campo la recepción

\footnotetext{
${ }^{1}$ Profesor en Filosofía y Ciencias de la Educación y Profesor en la cátedra de Epistemología I de la Facultad de Humanidades, Universidad Nacional de Catamarca (UNCA), San Fernando del Valle de Catamarca - Argentina. E-mail: naim_garnica@hotmail.com

Investigador del Instituto de Investigación en Teoría del Arte y Estética, UNCA. Licenciado en Filosofía, UNCA. Becario tipo I de CONICET.
}

http://dx.doi.org/10.1590/0101-3173.2018.v41n4.11.p205 
de la teoría francesa post-Mayo del ' 68 permitió pensar más allá de los cánones hegemónicos del heterosexismo. La lectura de Foucault, Lacan, Derrida, Deleuze y otros se constituye en la fuente de inspiración para las teorías que reflexionan sobre los cuerpos, las subjetividades, los géneros y las sexualidades. Desde el feminismo europeo hasta las teorías queer, aparecidas recientemente, el postestructuralismo pareciera ser el marco indispensable de reflexión y una cita obligada para pensar el problema político de la herencia de siglos anteriores. Dos ejemplos paradigmáticos de esta articulación entre teoría de género y post-estructuralismo son Judith Butler y Beatriz Preciado. En se reflejan las lecturas de Derrida y Foucault como preponderantes. Butler, siguiendo la filosofía del lenguaje de Derrida, la descripción histórica de la Historia de la sexualidad de Foucault y la crítica al feminismo de los años '70 construye su dispositivo de crítica denominado teoría de la performatividad. Por otra parte, Preciado, partiendo de las lecturas foucaultianas y derridianas, conforma su arqueología sobre la contra-sexualidad. Su punto de partida son los límites corporales del sistema heteronormativo inscriptos en los cuerpos. Lxs dxs autorxs reflejan en sus obras la presencia de un lenguaje estético subyacente al cual le otorgan efectividad política. La estética se abre paso en relación al concepto de género como un campo necesario de reflexión de su capacidad para modificar las estructuras culturales y políticas del statu quo. En consecuencia, el arte se afirma en su expresión y alcance ya no sólo como lo entendía la institución arte (idealismo y romanticismo) y la vanguardia, sino que adquiere la posibilidad de otorgarle sentido a aquello que no puede naturalizarse al orden tradicional. De algún modo, la estética en este plano asume una condición trasformadora y transfiguradora gracias a la soberanía que ejerce sobre otros discursos.

En consecuencia, en primer lugar, el objetivo general del trabajo es analizar las influencias y recepciones del post-estructuralismo en las lecturas de Judith Butler y Paul B. Preciado. A partir de esta exploración se podrían establecer las críticas de la teoría queer al feminismo a través de las imágenes y textualidades que propone el arte. La hipótesis general del trabajo supone que a partir de la recepción del postestructuralismo en los análisis de Butler y Preciado se podría reconocer en la estética la capacidad crítica y política que las teorías de la identidad sexual pretenden. A efectos de identificar estos legados nos detendremos en reconocer los aportes de Jacques Derrida en la obra de estxs autorxs. 
Debido a que la pretensión es rastrear la determinación que el postestructuralismo tuvo sobre ambxs autorxs y el modo en el que dicho proceso se manifiesta a partir de la estética, nos parece apropiado llevar a cabo una reconstrucción histórica de los conceptos. La pretensión de una metodología de este tipo accede a la construcción del campo histórico que dio lugar a la emergencia de las discusiones que abordaremos. En una primera instancia, nos detendremos en una revisión crítica de los textos de Derrida en orden a poner de relieve la recepción de los conceptos de este movimiento en las teorías de género. Los textos que tomaremos como centrales son Espolones, Corografías y Geschlecht: sexual diferencia, ontological difference. Los conceptos en los cuales haremos hincapié son: deconstrucción, sexualidad, historia, discurso, cuerpo, arte, huella, marca e iteración/iterabilidad. En segundo lugar, reconstruiremos rastros y semejanzas de tales conceptos en las teorías de género. En tercer lugar, se especificarán las formas de apropiación y recepción en ambos contextos. Finalmente, corresponde un relevamiento del valor de la instancia estética como una mediación fundamental en tal problemática. Esto permitirá reconstruir de qué modo las teorías de género leen el postestructuralismo y cuáles podrían ser los límites problemáticos en dicha recepción.

\section{ESTÉTICA, ARTE Y TRANSGRESIÓN}

En función de la relación entre la filosofía y el arte, la estética se constituye en un instrumento clave para poder reflexionar sobre la forma de deconstrucción de algunas categorías naturalizadas. La estética, como observamos en la literatura, el teatro o el cine, permite poner de relieve los dispositivos de transgresión de los modelos del imperativo heteronormativo de los cuerpos. La capacidad de romper la disposición occidental de los sujetos que posee la estética nos ayuda a construir nuevas miradas sobre las corporalidades que el sistema sexo-género quiere expulsar. Estas tendencias sostienen que el arte viene a ser una vía válida para recuperar aquello que, dentro de la cultura dominante, ha quedado sin articular en orden a un movimiento dialéctico que resucita la cultura. Si bien el posestructuralismo no se ocupa directamente del tema, da lugar a diversas relecturas de la obra de arte como despliegue infinito en el orden de la naturaleza. Los conceptos de huella, diferencia y ausencia, propios de la obra de Derrida, son materiales para una reasunción de la problemática del sujeto, la historia y la cultura. 
En este sentido, el arte se ha convertido para las teorías de género como una forma de expresión privilegiada de la deconstrucción o crítica de los valores tradicionales heterosexuales. A partir de la apropiación de conceptos artísticos como performatividad, transgresión, subversión, abyección o performance, las teorías de género intentan mostrar los límites impuestos por la cultura. Su capacidad subversiva radica, al igual que el arte, en poder transformar y criticar el orden establecido de los cuerpos, las sexualidades y la construcción de las subjetividades. Sin embargo, antes de llegar a ese punto de revisión veamos los intentos previos de construir una estética de la diferencia.

Ya el feminismo de la diferencia, al buscar elementos distintos o diferentes en la propia cultura patriarcal y androcéntrica, termina por estetizar posmodernamente aquellas categorías que pretendía impugnar. A partir de la lectura de Derrida y parte del pensamiento francés de los años '60, las pensadoras feministas elaboran una consideración mitologizada de aquello no aprehensible (lo imaginario) que sería lo femenino. El feminismo de la diferencia, en particular Irigaray, emplea a la historia colectiva de la humanidad para sostener que una de sus caras no participa de la racionalidad logocéntrica. Esto supone pensar en el carácter mitológico y legendario de una historia imaginaria que no descansa en los presupuestos de la razón ilustrada. En esa dimensión, el feminismo de la diferencia hace descansar gran parte de su oposición al falocentrismo dominante. Del algún modo, se podría considerar, como lo hace Kubissa, que autoras como ésta piensan que "la fuerza irreductible de lo femenino reside en la materialidad del cuerpo: lo corporal-femenino deviene en Irigaray contra-discurso del discurso masculino/patriarcal imperante." (KUBISSA, 2000, p. 237). La estética de la diferencia sería una denominación ambigua de la descripción que se pretende hacer del arte. Empleado originalmente por las feministas italianas como Murano, por una parte, como una adjetivación postestructuralista de la estética, por otra parte, este concepto intenta mostrar de qué modo se pueden encontrar relaciones entre estética y género a partir de algunas derivaciones del pensamiento de Derrida. No obstante, los límites del sujeto político del feminismo impiden pensar más allá de esa diferencia igualadora. Las críticas de Butler como de los estudios queer extenderán esas posibilidades del sujeto a consideraciones insospechadas para los parámetros liberales.

La presencia de Derrida en los estudios de Butler y Preciado pareciera estar implícita en sus lenguajes. Ambxs aceptan el postulado derridiano de que el lenguaje constituye una dimensión material donde los cuerpos encuen- 
tran la norma y, al mismo tiempo, la posibilidad de quiebre de tal norma. La transgresión del orden de los cuerpos se halla en la constitución misma de los cuerpos. Ya sea en la repetición performativa de Butler como en el paradigma prostético de Preciado, la consideración de la inestabilidad del lenguaje material de Derrida no puede evadirse. Siguiendo a Peggy Kamuf, se puede reconocer en Butler mucho más que en Preciado, un gesto claramente derridiano:

Just as clearly Butler's gesture is more complex if only because, in another sense, it also resembles or imitates Derrida's. More specifically, her marking of the collapse of the sex/gender distinction repeats a recognizable procedure of deconstruction that Derrida worked out, and, once again, very thoroughly in Of grammatology: the hierarchized terms of a binary opposition are reversed, the secondary or derived term is generalized as différance, which will have "produced" the opposition in the first place (although Derrida would never describe différance as "producing" differences or oppositions and still less as an "apparatus of production"). Likewise, in Butler's analysis, the distinction of sex from gender is said to be "produced" by the discursive condition identified with the second term; gender is thereby generalized as something like différance. Nevertheless, the generalization that Butler performs also leaves intact and undeconstructed the trait of secondary inscription. Thus, despite a certain recognizable outline of the deconstructive procedure, of which Butler makes such good use, a distance is also inscribed from the kind of thinking one associates foremost with Derrida. (KAMUF, 2002, p. 87). ${ }^{2}$

El vínculo que establece Kamuf entre Derrida y Butler se encuentra en la capacidad de advertir el cambio inherente a toda constitución lingüística, la cual no puede ser entendida ni como un efecto ni como una causa. En

${ }^{2}$ Hemos elegido colocar las citas en su idioma original dado que se podrían presentar demasiados giros al lector si sólo empleamos traducciones. Por ello, utilizamos los pies de página con la traducción espańola existente. En caso de que dicha traducción no exista, la traducción es nuestra.

"El gesto de Butler es complejo aunque sea porque, en otro sentido, también se parece o imita el de Derrida. Más específicamente, su marca del colapso de la distinción sex/gender repite un procedimiento reconocible de la deconstrucción que Derrida elaboró, y, una vez más, muy concienzudamente, en De la gramatología: los términos jerarquizados de una oposición binaria son revertidos, el término secundario o derivado es generalizado como différance, la cual habría producido la 'oposición' en primer lugar (aunque Derrida jamás describiría la difference como 'producir' diferencias u oposiciones y mucho menos como un 'aparato de producción'). Del mismo modo, en el análisis de Butler, la distinción de sexo y género sexual [sex from gender] se dice que es 'producida' por una condición discursiva identificada con el segundo término; gender, el género sexual, por lo tanto, es generalizado como algo parecido a la differance. Sin embargo, la generalización que Bulter lleva a cabo también deja intacto e indeconstruido el rasgo de la inscripción secundaria. Por lo tanto, a pesar de cierto esbozo del procedimiento deconstuctivo, del que Butler hace buen uso, también es inscrita una distancia de la clase de pensar que uno asocia en primer lugar con Derrida." (KAMUF, 2005, p. 121-122). 
Coreografias, una entrevista realizada por la feminista Christie V. McDonald, Derrida emplea una metáfora estética a los efectos de llevar a cabo una representación política del género. Si bien, en este caso, se trata de las mujeres, parece adecuarse a la descripción del concepto de género en términos más generales. Indica:

Perhaps woman does not have a history, not so much because of any notion of the "eternal feminine" but because all alone she can resist and step back from a certain history (precisely in order to dance) in which revolution, or at least the "concept" o revolution, is generally inscribed. That history is one of continuous progress, despite the revolutionary break - oriented in the case of the women's movement towards the reappropriation of woman's own essence, her own specific difference, oriented in short towards a notion of woman's truth. (DERRIDA, 1982, p. 68). ${ }^{3}$

La comparación con la danza muestra el modelo de soberanía del arte que Derrida provee a las teorías de género. Derrida invoca a la danza para descentrar el eje de las preguntas sobre el género. Al parecer, su propósito es mostrar que la historia del género puede contarse sin ese orden. Precisamente, esto es lo que significa "danzar de otro modo". Ello, supone transgredir el orden de la historia, en otras palabras, de qué modo se entiende la sexualidad.

Si el género pretende desestabilizar aquellas categorías que son ideológicamente dominantes respecto de las esferas políticas, entonces, éste debe asumir una forma del arte capaz de desestabilizar dichas categorías. Así, la posibilidad del arte como discurso soberano sobre otras esferas comienza a ser empleado como recurso ontológico y político por parte de los estudios de género. La performance, el arte de la cirugía, la danza o la transformación, perecieran constituirse en los modelos de discurso capaces de modificar la ontología de la identidad del sujeto. Asimismo, cuestionan las categorías dominantes del discurso político de Occidente. En este punto, podría advertirse cierta disposición a una extralimitación de las consideraciones del arte en beneficio de alcanzar un objetivo como la crítica de lo establecido. Como ya lo habría advertido Bubner en su programático texto "Sobre las condiciones de la estética actual” a mediados de los años '70, la experiencia estética pereciera fundar

3 'Puede ser que la mujer no tenga historia, no a causa de un 'eterno femenino', sino porque se puede, solo, sola, resistir, separarse (para danzar, precisamente) de cierta historia en la que se inscribe en general la revolución o, al menos, su 'concepto', la historia como progreso continuo, a pesar de la ruptura revolucionaria, historia aquí orientada por el movimiento de la mujer hacia la reapropiación de su propia esencia, de su propia diferencia, hacia su 'verdad'.” (DERRIDA; MCDONALD, 2008, p. 160). 
un modelo soberano sobre otros discursos escudado en su propia autonomía, la cual guarda su propio cuestionamiento. Pereciera, por un lado, apelar al modelo romántico de que el arte sólo puede ser criticado por el arte mismo, replegando el discurso sobre sí mismo, a fines de protegerse de otros discursos. Por otro lado, se le exige al arte la necesidad de alcanzar los efectos políticos de una deconstrucción de las categorías que regulan los cuerpos, el género y la sexualidad. Por ejemplo, para Preciado el género logra conseguir esta danza mediante la plasticidad del cuerpo. En el Manifiesto contra-sexual dice:

El género no es simplemente performativo (es decir, un efecto de las prácticas culturales lingüístico-discursivas) como habría querido Judith Butler. El género es ante todo prostético, es decir, no se da sino en la materialidad de los cuerpos. Es puramente construido y al mismo tiempo enteramente orgánico. Escapa a las falsas dicotomías metafísicas entre el cuerpo y el alma, la forma y la materia. El género se parece al dildo. Porque los dos pasan de la imitación. Su plasticidad carnal desestabiliza la distinción entre lo imitado y el imitador, entre la verdad y la representación de la verdad, entre la referencia y el referente, entre la naturaleza y el artificio, entre los órganos sexuales y las prácticas del sexo. El género podría resultar una tecnología sofisticada que fabrica cuerpos sexuales. (PRECIADO, 2002, p. 25).

En este caso en particular, la danza aparece como aquella forma indispensable que hace girar de otro modo las narraciones sobre el género femenino. De ese modo, se forman las posibilidades insospechadas de reinvención de los cuerpos que Butler y Preciado imaginan.

\section{ESTÉTICA Y GÉNERO}

Ahora bien, detengámonos en esa posibilidad irreductible que la danza presenta para frustrar cualquier asignación referencial del género. Precisamente, será esto lo que Butler y Preciado explotaran en sus respectivas críticas al heteronormativismo. Dice Derrida:

This is very rare, if it is not impossible and presents itself only in the form of the most unforeseeable and most innocent of chances. The most innocent of dances would thwart the assignation a residence, escape those residences under surveillance; the dance changes place and above all changes places. In its wake they can no longer be recognized. The joyous disturbance of certain women's movements, and of some women in particular, has actually brought with it the chance for a certain risky turbulence in the assigning of places with in our small European space (I am not speaking 
of a more ample upheaval en route to world wide application) Is one then going to start all over again making maps, topographics, etc.? Distributing sexual identity cards. (DERRIDA, 1982, p. 69). ${ }^{4}$

\section{La danza o coreografía, bajo el análisis de Derrida, parece ofrecerle a} Butler y Preciado la posibilidad de una permanente deconstrucción de la identidad en términos de una diferencia ontológica constitutiva de la subjetividad. El famoso lema de "abrir las posibilidades" de la Butler de Gender trouble aparece a través de la soberanía estética de la danza de Derrida en tanto multiplica las formas de representar, ver, entender y desplazar al género (BUTLER, 1990). Derrida, incluso, radicaliza lo anteriormente expuesto:

The relationship would not be a-sexual, far from it, but would be sexual otherwise: beyond the binary difference that governs the decorum of all codes, beyond the opposition feminine/masculine, beyond bisexuality as well, beyond homosexuality and heterosexuality which come to the same thing. As I dream of saving the chance that this question offers I would like to believe in the multiplicity of sexually marked voices. I would like to believe in the masses, this indeterminable number of blended voices, this mobile of non-identified sexual marks whose choreography can carry, divide, multiply the body of each "individual", whether he be classified as "man" or as "woman" according to the criteria of usage. (DERRIDA, 1982, p. 76).

Según Anne Berger en su ensayo "Sexuar las diferencias", la danza supone el paradigma por excelencia para pensar la diferencia sexual en términos derridianos. Berger sostiene que Derrida lo hace, de forma sorprendente, in-

\footnotetext{
4 "La danza más inocente ya no daría juego a arrestos domiciliarios, como se dice en francés, a residencias vigiladas, esa danza cambia de lugar, cambia sobre todo los lugares. Tras su paso, ya no se reconoce el sitio. La alegre perturbación que tales movimientos de mujeres, y también algunas mujeres singulares, han aportado a nuestro pequeño espacio europeo (ya no hablo de un seísmo más amplio y en vías de mundialización), ‘acaso no ofrece precisamente la ocasión para alguna turbulencia aleatoria en la asignación de lugares? Entonces, ¿volveremos a construir esos documentos geográficos, topográficos, etc.? ¿'A distribuir documentos de identidad sexual?” (DERRIDA, 2008, p. 161).

5 “'Relación, entonces, ya no asexuada, ni mucho menos, sino sexuada de otra manera, más allá de la diferencia binaria que gobierna la conveniencia de todos los códigos, más allá de la oposición femenino/ masculino, como también más allá de la bisexualidad, de la homosexualidad o de la heterosexualidad que resultan ser lo mismo? Sońando con salvar al menos la ocasión de plantear esa pregunta, querría creer en la multiplicidad de voces sexualmente marcadas, en ese número indeterminable de voces entrelazadas, en ese móvil de marcas sexuales no identificadas cuya coreografía puede arrastrar el cuerpo de cada 'individuo', atravesarlo, dividirlo, multiplicarlo, ya esté clasificado como 'hombre' o como 'mujer' según los criterios al uso.” (DERRIDA, 2008, p. 171).
} 
vitándola e invitándonos a meditar sobre los vínculos entre danza, différance, lectura y diferencia sexual. Tomando el ejemplo de una "mujer inconformista" (Emma Goldman) citada por otra (McDonald en la entrevista antes mencionada), el pensador francés "dice" lo que ella dice que ella dijo (y decir lo que el otro dice, es decir, citar, mencionar consciente o inconscientemente es lo que Derrida afirma que hacemos todos, continuamente, por ejemplo, en cuanto decimos: "dancemos") (BERGER, 2008). La "danza", no constituye un concepto de la historia de la filosofía, su actividad podría desestabilizar precisamente el propio lenguaje. Según parece, la dimensión estética que Preciado y Butler asumen necesita evocar conceptos irreconocibles que podrían extraviarse y extraviarnos.

El concepto de danza es valorado como una forma de representar la deconstrucción, el diferimiento o una re-escritura que ni el propio Derrida conoce. Esta forma de escritura a-gramatical realiza un juego etimológico que habilita a pensar en las diversas posibilidades que se abren en ese movimiento sin un efecto determinando. Una posible consecuencia que se extrae de esa metáfora, permite pensar en la posibilidad de una diseminación del lenguaje que las identidades de género llevan a cabo. A su vez, se puede observar la dificultad política que ese movimiento plantea a los firmes posicionamientos políticos que los colectivos feministas como homosexuales han llevado a cabo en sus luchas.

Sin embargo, la discusión de Derrida es ontológica en primera instancia, para luego asumir sus consecuencias políticas. Derrida no sostiene que la identidad sexual no exista debido al diferimiento permanente, en este marco, Butler, en particular, y Preciado en general, toman el modelo de corrimiento de la identidad para poder explicar la ausencia de un sujeto liberal auto-controlado. Los desplazamientos de la danza intentan representar cómo la subjetividad no encuentra el lugar seguro desde donde constituir su identidad. Berger cree importante no olvidar que "no se pare el movimiento, que la posibilidad de danzar quede abierta, a la vez que se preserva la posibilidad de posición, o de estasis." (BERGER, 2008, p.176).

Así, para Derrida, tanto la danza como el concepto de género comparten una condición que es estructural a todo lenguaje: la condición de inestabilidad. La condición inestable de toda estructura, es decir, su forma danzante permite identificar una diferencia estética necesaria para pensar los quiebres de la identidad de género. La explicación de Berger es, tal vez, la más representativa de estas tendencias que logran estetizar sus descripciones: "La lectura es 
o debería ser un proceso o un movimiento abierto e infinito: una especie de danza." (BERGER, 2008, p. 176).

En consecuencia, la lectura del género queda establecida en un movimiento permanente que no permite recocer una identidad pre-establecida. La interpretación de Berger, en este sentido, sostiene que "se podría decir que, para Derrida, "sexo" (la palabra y lo que designa en francés y en inglés), los cortes de los sexos (aunque sean, como los sueña Derrida, "indeterminables" e "innumerables"), son una condición tanto del amor como de la danza" (BERGER, 2008, p. 182). Incluso, Berger no guarda ningún reproche sobre la comparación entre danza y género. Al inicio de su ensayo confirma la relación entre danza y diferencia sexual que se deja entrever en las lecturas de Preciado y Butler sobre Derrida:

¿Qué tiene que ver la "diferencia sexual" con "danzar" y "leer"? Yo diría que la invitación a danzar de Derrida es también, necesariamente, una invitación a reescribir y reinscibir la "diferencia sexual" como (y en) "diferancias sexuales" (con una "a" y en plural), es decir, como una multiplicidad de pasos divididos que se resisten a formalizaciones estables, una danza que difiere y agita a los sujetos y los forma en el/los movimiento(s), que los empuja hacia y lejos el uno del otro. (BERGER, 2008, p. 177).

Estos planteos aparecen en los ensayos de Derrida sobre su crítica al Dasein asexuado de Heidegger y la consideración de la preocupación secundaria que Levinas le atribuye a la diferencia sexual. En ellos, como así también en Espolones, se puede identificar algunos puntos seguidos por las teorías de género. La idea de deconstrucción, en esa dirección, no es la impugnación de esas categorías, sino la multiplicación de las posibilidades del género. En textos como Vida precaria o Marcos de guerra Butler emplea el concepto de iterabilidad para referirse a las posibilidades insondables de la democracia radical, llevando a cabo una operación similar a la de Derrida para pensar la literatura. La literatura, juzga Derrida, posee constantes recontextualizaciones por las repeticiones y abre alteraciones, las cuales favorecen la posibilidad de nuevos marcos que se encuentran radicalmente distanciados de aquellos que les permitieron su existencia. La iterabiidad que Derrida supone para la literatura es transferida al concepto de democracia y empleada a los efectos de pensar la exclusión de las vidas, como también de su reconocimiento por medio de la melancolía y el duelo-luto. 


\section{Refiriéndose a la diferencia sexual que atraviesa toda descripción con- ceptual Derrida plantea la siguiente problemática:}

The concept of the concept, along with the entire system that attends it, belongs to a prescriptive order. It is that order that a problematic of woman and a problematic of difference, as sexual difference, should disrupt along the way. Moreover, I am not sure that "phase two" marks a split with "phase one", a split whose form would be a cut along an indivisible line. The relationship between these two phases doubtless has another structure. I spoke of two distinct phases for the sake of clarity, but the relationship of one phase to another is marked less by conceptual determinations (that is, where a new concept follows an archaic one) than by a transformation $r$ general deformation of logic; such transformations or deformations mark the "logical" element or environment itself by moving, for example, beyond the "positional" (difference determined as opposition, whether or not dialectically). This movement is of great consequence for the discussion here, even if my formulation is apparently abstract and disembodied. One could, I think, demonstrate this: when sexual difference is determined by opposition in the dialectic as sense (according to the Hegelian movement of speculative dialectics which remains so powerful even beyond Hegel's text), one appears to set off "the war between the sexes"; but one precipitate the end with victory going to the masculines ex. The determination of sexual difference in opposition is destined, designed, in truth, for truth; it is so in order to erase sexual difference. The dialwereectical opposition neutralizes or supersedes [Hegel's term Aufhebung carries with it both the sense of conserving and negating. (DERRIDA, 1982, p. 72). ${ }^{6}$

\section{En consecuencia, Derrida radicaliza su postura:}

\footnotetext{
6 "Con todo su sistema, el concepto de concepto pertenece a esa ordenación que una problemática de la mujer y de la diferencia como diferencia sexual debería alterar a su paso. Además, no estoy seguro de que la 'fase 2' marque una ruptura con la 'fase 1', una ruptura bajo la forma de un corte a lo largo de un trazo indivisible. La relación de esas dos fases tiene sin duda otra estructura. Por comodidad expositiva, hablé de fases distintas, pero la relación de una fase con la otra se marca menos por determinaciones conceptuales (un nuevo concepto sucede a un concepto arcaico) que por una transformación o una deformación general de la lógica, del elemento o del mismo medio 'lógico', por ejemplo, por el paso más allá de lo 'posicional' (diferencia determinada como oposición, dialécticamente o no). Ello tiene grandes consecuencias para lo que estamos hablando, incluso si lo estoy formulando de una manera vacía y desencarnada en apariencia. Podríamos, creo, demostrarlo: cuando se determina la diferencia sexual a partir de la oposición en el sentido dialéctico, según el movimiento hegeliano de la dialéctica especulativa cuya necesidad sigue siendo muy potente mucho más allá del texto de Hegel, parece que se desencadene una guerra de sexos; aunque precipitamos su fin con la victoria del sexo masculino. La determinación de la diferencia a partir de la oposición está destinada en verdad, a la verdad, a borrar la diferencia sexual. La oposición dialéctica neutraliza o 'supera' [relève] la diferencia." (DERRIDA, 2008, p. 165).
} 
And what if the "wife" or the "mother" -whom you seem sure of being able to dissociate- were figures for this homosexual dialectic? I am referring now to your question on the "representation" of woman and such "loss" as might occur in the passage from man's rib to the womb of woman, the passage from the spouse, you say, to the mother. Why is it necessary to choose, and why only these two possibilities, these two "places" assuming that one can really dissociate them? (DERRIDA, 1982, p. 72). ${ }^{7}$

Sin embargo, la diferencia sexual no es el problema nuclear para Derrida, sino el concepto de Humanidad (Espíritu) que subyace a esa diferencia. Recurriendo al análisis ontológico del Dasein de Ser y tiempo de Heidegger, Derrida propone pensar la diferencia en términos de diferencia ontológica y no a modo de diferencia sexual. ${ }^{8}$

Finalmente, una imagen todavía más ejemplar de esta relación entre género y danza que establece una estética de la diferencia, es la que ofrece la teoría performativa explicada bajo la figura de la Drag queen. En las páginas finales a Gender trouble, Butler se refiere a un mecanismo crítico de la identidad sexual autofundante mediante la imagen de la teatralidad danzante de la Drag. En ese recurso se cifra esta forma de estética que busca emplear prácticas artísticas a los fines de cuestionar otros discursos, por ejemplo, el de la sexualidad. Esto no es tan distante de la función asignada en el modelo de la soberanía del arte de la deconstrucción. Cuando Derrida pregunta “¿Qué sería la danza?” y responde con otro interrogante "¿habría danza si no intercambiáramos ahí los sexos en un número indeterminado, con ritmos muy variables?”, muestra el efecto violento del arte sobre otros discursos de la racionalidad. No obstante, no puede descuidarse el sentido ampliado que el concepto de soberanía tiene

\footnotetext{
7 “¿Y si 'la esposa’ o 'la madre’ - que usted parece estar muy segura de poder disociar - fueran todavía figuras de esa dialéctica homosexual? Me estoy refiriendo ahora a su pregunta sobre la 'representación' de la mujer y sobre tal 'pérdida' en el paso de la costilla del hombre a la vagina de la mujer, de la esposa, como usted dice, a la madre. ¿Por qué habría que escoger, y solamente entre esas dos posibilidades, esos dos 'lugares', suponiendo de nuevo que pudiéramos verdaderamente disociarlos?" (DERRIDA, 2008, p. 166).

${ }^{8}$ En Testo yonki Preciado considera algo similar pero con mayor especificidad temporal: "El género del siglo XXI funciona como un dispositivo abstracto de subjetivación técnica: se pega, se corta, se desplaza, se cita, se imita, se traga, se inyecta, se injerta, se digitaliza, se copia, se diseña, se compra, se vende, se modifica, se hipoteca, se transfiere, se download, se aplica, se transcribe, se falsifica, se ejecuta, se certifica, se permuta, se dosifica, se suministra, se extrae, se contrae, se sustrae, se niega, se reniega, se traiciona, muta." (PRECIADO, 2008, p. 88).
} 


\section{en Derrida, en particular, si se lo pone en relación a la política9?. Pese a ello, autoras como Kamuf les interesa remarcar que si bien Derrida no usa el térmi- no género/gender permite pensar la diferencia del lenguaje para designar los géneros. Indica:}

Derrida has never written the word gender, it was in order to call attention right away to the inscription of something other, perhaps an other (than) sexual difference on the body of his language. Instead of a general reproduction of gender, by that name and therefore only in one particular language, there would be the inscription in a different language of another "generalization." But if gender is indeed being generalized in other names by that writing, then this leaves open the possibility that anything or everything Derrida has written can be taken to refer its reader somewhere or somehow to (a) sexual difference. (KAMUF, 2001, p. 88). ${ }^{10}$

${ }^{9}$ En The dictionary of Derrida se lee: "Soberanía: Mientras que Derrida está siempre interesado en las operaciones altamente singulares y específicas del poder, la política, o la ley, hay un sentido más general del término 'soberanía' en su escritura que va un poco más allá de su uso común limitado, por ejemplo, en relación a las formas históricas tomadas por la monarquía o la autodeterminación de un Estado-nación. (Estas son, sin embargo, de gran interés para Derrida, y nunca pueden dejarse de lado cuando se pregunta acerca de la soberanía.) La cuestión de la soberanía surge siempre que sea una entidad que se imaginaba en términos de su poder de dominio (incluyendo el auto-dominio), siempre que se considere capaz de auto-expresión autorizada, o donde la unidad ostensible, la propia identidad y la autosuficiencia (la presencia supuestamente indivisible) de un ser se impone forzosamente a expensas de la diferencia y el otro. Para Derrida, recordemos, différance es, de hecho, constitutivo o 'originario'. Sin embargo, siempre es reprimido por la tradición metafísica que tiene presencia (no auto-diferencia-) para ser soberana. Por lo tanto, la soberanía es deconstruible - su dominio, aparentemente incuestionable, está en todas partes atravesada por un resto no-presente: différance, vestigios, suplemento, archi-escritura, el otro. Esto puede ilustrarse mediante la lectura de Derrida de la Declaración de la Independencia [...] A lo largo de muchos de los escritos de Derrida, la cuestión de la soberanía se pone al lado de los debates de la ciudadanía, la apatrida, la forma histórica de la polis y el Estado moderno, la importancia y las limitaciones del derecho internacional, y el significado de la biopolítica y la vida animal. En El cosmopolitismo y el perdón, por ejemplo, lo ilimitado o incondicional 'bienvenido' exigió de la hospitalidad en su sentido fundamental, está en primer plano con el fin de provocar la reflexión y de hecho la acción con respecto a las formas históricas de derecho y el derecho asociado a la soberanía. Al adoptar este enfoque, Derrida no imagina por un momento que sería posible en la práctica - o incluso preferible, necesariamente - para barrer las fronteras y los poderes de soberanía. En cambio, su apelación a la 'incondicional' nos enfrenta a una responsabilidad continua de pensar en modificar o transformándolos. [...] Lejos de quitarle poder a la democracia, entonces, esta división irresoluble significa que la democracia sigue siendo irreductible al poder soberano y la presencia.)” (MORGAN WORTHAM, 2010, p. 190-192, traducción es nuestra).

10 "Derrida no ha escrito nunca la palabra gender, fue a fin de llamar inmediatamente la atención sobre la inscripción de algo otro, tal vez otro (que) la diferencia sexual en el cuerpo de su lengua. En lugar de una reproducción general del gender, por ese nombre y por ende únicamente en un lenguaje particular, existiría la inscripción de otra generalización en un lenguaje diferente. [...] esto abre la posibilidad de que cualquier cosa o todo lo que Derrida ha escrito pueda ser tomado para remitir a su lector, en alguna parte o de alguna manera, a (la) diferencia sexual.” (KAMUF, 2005, p. 123). 
Esto, encuentra su sentido en el concepto de diseminación empleado por Derrida en dos direcciones co-implicadas. Por una parte, porque diseminación remite a una imposibilidad del sentido asignado por el imperativo masculino (la seminación) evitando la reproducción. Pero, por otro lado, mantiene un sentido no privativo, dado que diseminación también remite al esparcimiento. En palabras de Kamuf: "For Derrida has therefore also to be heard as a calculated, strategic displacement of the term reproduction, above all sexual reproduction, and a fortiori of the term Butler privileges: production, as in 'apparatus of production'." (KAMUF, 2001, p. 88). ${ }^{11}$

La diseminación, en esa doble operación, logra referirse a una sexualización del lenguaje, algo que, por lo general, el discurso filosófico neutraliza o homogeniza. La invocación a Derrida, por parte de Butler, permite especificar la posibilidad de tematizar la diferencia y, al mismo tiempo, introduce la complejidad de reconocer la diferencia sexual que recorre toda política. A los efectos de equilibrar este problema, tanto Butler como Preciado, recurren a metaforizar la sexualidad y pretenden volver comprensible los procesos de subjetivación sexual mediante la apelación a la estética. Tanto la teoría performativa como la teoría prostética de Preciado recurren a imágenes artísticas para describir los procesos por los cuales es posible poder pensar la diferencia sexual, como asimismo, los procesos de cambio que la sexualidad mantiene sin ontologizar al sujeto.

\section{A MODO DE CIERRE: LA ESTÉTICA ENTRE SOBERANÍA E IDEOLOGÍA}

La apropiación de la propuesta derridiana por parte de Butler y Preciado, parece evidenciar el intento de resolver problemas filosóficos, políticos y ontológicos en problemas artísticos, reduciendo en la verdad del arte verdades de otra índole. De alguna manera, la lectura de Butler y Preciado en su estética de la diferencia sexual, en términos ontológicos, puede entenderse como una estética heterónoma. Tal concepto, puede ser entendido siguiendo la crítica de Bubner, como una forma de tutelaje del arte mediante el lenguaje filosófico. El arte, en consecuencia, se encuentra en la obligación de revelar verdades más profundas, en este caso, de una diferencia sexual radical que se mueve como la danza. Incluso, debería develar verdades filosóficas que la filosofía misma

\footnotetext{
11 "Para Derrida, por lo tanto, tiene que oírse también como un desplazamiento calculado, estratégico del término reproducción, por encima de toda reproducción sexual, y a fortiori del término que Butler privilegia: producción, como aparato de producción." (KAMUF, 2005, p. 84).
} 
ya no se atreve a hacerlo. Lo que inicialmente parece ser una revalorización del arte, es para Bubner su devaluación, por reducir el arte a la filosofía. De ese modo, el arte pierde su propia lógica, dado que el peligro con estas estéticas heterónomas radica en la subordinación del arte a la filosofía (BUBNER, 2010).

Ya Christoph Menke advertía en su texto La soberanía del arte (1997) que el modelo de soberanía de Derrida implicaba una forma de colocar al arte sobre otros discursos a los fines de resolver problemas extra-estéticos. Indica Menke sobre la pretensión de Derrida:

La marginación de la experiencia estética [...] no significa, según Derrida, más que la complicidad de la estética tradicional con la metafísica, es decir, en el lenguaje derridiano, con la reconstrucción de nuestros discursos con vistas a su funcionamiento eficaz. Por eso entiende Derrida el "reconocimiento" del arte en la estética como su sometimiento a una forma de discurso entre otros, en el que pierde su pretensión subversiva [...] Derrida llama sometida o servil a la concepción del arte que lo rebaja a una forma limitada de discurso, frente a su contenido soberano. (MENKE, 1997, p. 192).

Por el contrario, para Derrida y como se puede ver en el modelo estético de Butler y Preciado, la soberanía está dada en la medida en que el arte es pensado como soberano "porque sobrepasa los deseos del sentido que determinan los discursos no estéticos. Considerar el arte en su soberanía, significa no eludir ni rechazar el peligro de no tener sentido, sino asumirlo y preservarlo. Es servil el rechazo de tal riesgo, y soberano su asunción." (MENKE, 1997, p. 192). Menke sostiene que la deconstrucción no puede limitarse sólo a una mera descripción antifundacionalista, sino que ella "significa un análisis del funcionamiento del lenguaje que, por analogía con la experiencia estética de la negatividad, descubre en ese funcionamiento mismo la raíz de su fracaso." (MENKE, 1997, p. 216). Precisamente, esa inestabilidad del lenguaje es la que muestra las teorías de género apropiándose del modelo de la soberanía estética.

Por otra parte, y para finalizar, podemos observar que los planteamientos antes indicados que se encuentran tanto en Butler como en Preciado, parecieran caer en la descripción que Paul De Man (1998) hace del concepto de "ideología estética". La posibilidad de que un mecanismo estético como la danza brinde o asegure un proceso político de cuestionamiento, se adecua 
a las características del concepto de ideología estética que De Man identifica en su lectura de la danza en Kleist, como también, en el concepto de juego de Schiller. Sin embargo, la función otorgada al arte como forma de crítica subversiva puede cuestionarse $\mathrm{o}$, al menos, nos provoca algunos interrogantes: ¿puede el arte llevar a cabo una crítica contra otras esferas sin renunciar a su autonomía? ¿De qué modo el arte provoca una subversión de otras esferas de la razón sin volverse una promesa de superación? ¿Es el arte la última esfera de la racionalidad donde las esperanzas de cambio y transformación pueden encontrarse? ¿¿No es la estética también, como sostenía Paul De Man, una forma de sustitución categorial (metafórica) que reintroduce de otro modo las mismas racionalidades que se pretenden subvertir? Estos interrogantes, como también, los postulados sobre la identidad del sujeto en las teorías de género todavía parecen encontrarse en plena discusión.

GARNICA, N. Post-structuralism and aesthetics: between art and gender. Trans/form/ação, Marília, v. 41, n. 4, p. 205-222, Out./Dez., 2018.

\begin{abstract}
The general objective of this article is to analyze the influence of post-structuralism in the thought of Judith Butler and Paul B. Preciado. It is argued that queer theory's critique of feminism can be established through the images and textualities proposed by art; furthermore, on the basis of the influence of poststructuralism in Butler's and Preciado's analyses, one can recognize in aesthetics the critical and political capacity that theories of sexual identity intend to achieve. In order to identify these influences, we discuss the contributions of the ideas Jacques Derrida to the work of these authors. In conclusion, we raise some criticisms that warn of the possible limits of gender theories in the plane of aesthetics.
\end{abstract}

KeYwords: Aesthetics. Gender. Critique. Art. Dance.

\title{
REFERENCIAS
}

BERGER, A. Sexuar las diferencias. Lectora, n. 14, p. 173-187, 2008.

Sobre algunas condiciones de la estética actual. In: Acción, historia y orden institucional: ensayos de filosofía práctica y una reflexión sobre estética. Argentina: FCE, 2010. p. 357-410. 
Mecanismos psíquicos del poder: teorías sobre la sujeción. Madrid: Cátedra, 2001. (1993 1ra Edición).

. Vida precaria: el poder de la violencia y el duelo. Bs. As: Paidós, 2006.

- El género en disputa: el feminismo y la subversión de la identidad. Barcelona: Paidós, 2007. (1990 1ra Edición).

. Marcos de guerra: las vidas lloradas. Bs. As: Paidós, 2010.

CRITCHLEY, S. The ethics of deconstruction: Derrida and Levinas. Oxford Press, Polity, 1992.

DE MAN, P. La ideología estética. Madrid: Cátedra, 1998.

DERRIDA, J. Spurs/Eperons. Translation by B. Harlow. Chicago: University of Chicago Press, 1979. (Traducción castellana: Espolones. Valencia: Pre-Textos. 1981).

. Geschlecht. Sexual difference, ontological difference. Trans. R Berezdivin. Research in Phenomenology, n. 13, p. 65-83, 1983.

.; MCDONALD, C. Interview: Choreographies. Diacritics: Cherchez la Femme Feminist Critique/Feminine Text, v. 12, n. 2, p. 66-76, Summer, 1982. (Traducción castellana DERRIDA, J.; MCDONALD, C. V. Coreografías. Lectora, n. 14, p. 157-172, 1995).

GROSZ, E. Ontology and equivocation: Derrida's politics of sexual difference. Diacritics, v. 25, n. 2, p. 114-124, Verano, 1995.

KAMUF, P. Derrida and gender: the other sexual difference. In: COHEN, T. Derrida and humanities: a critical reader. UK: Cambridge University Press, 2001. p. 82-107. (Traducción castellana: COHEN, T. Jacques Derrida y las humanidades. Traducción Ariel Dilon. México; Buenos Aires: Siglo XXI, 2005).

KUBISSA, L. De discursos estéticos, sustituciones categoriales y otras operaciones simbólicas: en torno a la filosofía del feminismo de la diferencia. In: AMOROS, C. (Ed.). Feminismo y filosofía. Espańa: Edit. Síntesis, 2000. p. 213-254.

MENKE, C. La soberania del arte. Madrid: Visor, 1997.

. Estética y negatividad. México: FCE, 2011.

MORGAN WORTHAM, S. The Derrida Dictionary. London: Continuum, 2010.

PRECIADO, B. Manifiesto contra-sexual. Barcelona: Anagrama, 2002.

. Testo yonki. España: Espasa-Calpe, 2008.

Recebido: 02/02/2016

Aceito: 08/09/2017 
GARNICA, N. 